\title{
Study on the Effectiveness of Single Lightning Rods by a Fractal Approach
}

\author{
Xuewei Zhang, Lin Dong, Jinliang He*, Shuiming Chen and Rong Zeng
}

State Key Lab of Power Systems, Department of Electrical Engineering, Tsinghua University, Beijing 100084, China

\begin{abstract}
Lightning rod is widely used in lightning protection systems. It is of practical interest to analyze the protective performance of a lightning rod taking into consideration some stochastic behaviors of the lightning process. The effectiveness of a single lightning rod is investigated in this paper using a fractal growth approach based on dielectric breakdwon model (DBM). The effective interception area, the strike distribution on rod and the protection zone of single lightning rods of various heights are calculated. Comparing our results with those of the rolling sphere method, it is shown that, the fractal approach is able to make reasonable evaluation of the lightning protective performance. New features such as the inherent vulnerability of the rod to lateral strikes and the division of the protection zone into three regions are also presented.
\end{abstract}

Keywords: Lightning rod, fractal simulation, interception area, strike distribution, protection zone.

\section{INTRODUCTION}

Franklin's invention of lightning rod is effective in preventing damages to protected structures from lightning strikes. Till today for more than 200 years, the lightning rod has been the most widely used air terminal of lightning protection systems. Unfortunately, lightning rods can not always function perfectly. There have been reports on unexpected shielding failures, which, as we know, are often due to the randomness of lightning phenomenon. For example, several direct-strikes to the transformer substations took place in the power grid of southern China. However, when designing a lightning protection system, the protection angle method, the rolling sphere method and the mesh method are used to evaluate the protection zone of a lightning rod [1], all of which fall short of considerations on stochastic behaviors of the lightning process. It is therefore of practical interest to investigate the effectiveness of lightning rods by means of dynamic simulation of lightning strikes including stochasity.

Before that, it is necessary to have a basic understanding of the phenomenology of the lightning attachment process. Detailed discussions can be found in Ref. [2]. It is also essential to take into account the probabilistic nature and branching, tortuous characteristics of lightning discharges. Numerical model of lightning leader process based on fractal growth named dielectric breakdown model (DBM) has been developed and verified in Ref. [3-9], which present more realistic lightning channels. Nevertheless, though its potential for practical applications has been demonstrated in Ref. [4], the fractal simulation of lightning has mostly been used as a descriptive or visualization tool of lightning processes and channels rather than a predictor or analyzer in lightning protection engineering. As an attempt to convert this methodology to practical utility, in the present work we calculate the interception area, the strike distribution and the protection zone of single lightning rods of various heights using the

*Address correspondence to this author at the State Key Lab of Power Systems, Department of Electrical Engineering, Tsinghua University, Beijing 100084, China; Tel: +86-10-62775585; E-mail: hejl@tsinghua.edu.cn fractal approach. By comparing our results with those of the rolling sphere method, it is seen that the fractal approach is competent in reasonably evaluating the effectiveness of single lightning rods.

The rest of this paper is organized as follows. In Section 2 , the algorithm, configuration and parameter settings of our simulations are briefly introduced. In Section 3, the interception area and strike distribution of a single lightning rod are investigated. In Section 4, we discuss the protection zone of a single 40-meter lightning rod in detail and make comparison between the fractal approach and the rolling sphere method; more cases with different rod heights are calculated, too. Some conclusive remarks are drawn in Section 5.

\section{FRACTAL SIMULATION OF LIGHTNING LEADER}

\section{A. Overview of DBM}

We introduce the algorithm of DBM in the context of 2D planar discharge. The discharge starts from somewhere on the upper boundary and develops toward the lower boundary. Within the simulation boundary, under the quasi-static approximation, the potential of the electric field is depicted by Poisson's equation. Taking the lower boundary and the discharge channel as the first-type boundary condition, i. e. the potentials at the points in these areas are fixed, it is then unnecessary to consider the ambient charge because we have included its effect in the boundary conditions. Hence, the potential $\varphi_{i j}$ at discrete point $(i, j)$ can be obtained by solving the discretized Laplace equation (1) (with exception that applying free boundary condition at other boundaries):

$\varphi_{i j}=\frac{1}{4}\left(\varphi_{i+1, j}+\varphi_{i-1, j}+\varphi_{i, j+1}+\varphi_{i, j-1}\right)$

A single simulation step of discharge process is as follows: suppose a point $\left(i, j^{\prime}\right)$ that is not located in but neighboring to the channel and the mean field intensity between this point and a nearby channel point $(i, j)$ exceeds a critical value, as shown in Eq. (2):

$E_{i^{\prime} j^{\prime}, i j} \equiv\left|\left(\varphi_{i^{\prime} j^{\prime}}-\varphi_{i j}\right) / d_{i^{\prime} j^{\prime}, i j}\right| \geq E_{c}$ 
where $d_{i{ }^{\prime}{ }^{\prime}, i j}$ denotes the distance in between. This point is a possible discharge point of the next step. The probability $p$ for a possible discharge point to become a new channel point is related to the local field intensity, which can be expressed as Eq. (3):

$$
p\left(i, j \rightarrow i^{\prime}, j^{\prime}\right)=\frac{E_{i^{\prime} j^{\prime}, i j} \eta}{\sum E_{i^{\prime} j^{\prime}, i j} \eta}
$$

where $(i, j)$ and $\left(i, j^{\prime}\right)$ are a channel point and a possible discharge point respectively, $\eta$ is called the development probability index. The probability for the channel to develop from $(i, j)$ to $\left(i, j^{\prime}\right)$ is proportional to the $\eta$ th power of the mean field between the two points. The denominator at right side of Eq. (3) is summation over all combinations of $(i, j) \mathrm{s}$ and $\left(i, j^{\prime}\right)$ s. Note that such a combination can be built from every channel point and every possible discharge point neighboring to the channel point.

According to the calculated probability we randomly select the discharge direction for the next step. If the new channel section is from $(i, j)$ to $\left(i^{\prime}, j^{\prime}\right)$, assuming that the electric field along the channel is $E_{c h}$, we need to update the potential at point $\left(i, j^{\prime}\right)$ as shown in Eq. (4):

$\tilde{\varphi}_{i^{\prime} j^{\prime}}=\varphi_{i j}-E_{c h} d_{i^{\prime} j^{\prime}, i j}$

Then we begin the next step of simulation and repeat this until the channel connects the upper and lower boundaries, which means that the gap has broken down.

\section{B. Configuration and Procedures of Fractal Simulation}

In this paper we only investigate the protective performance of a single rod against downward negative lightning. In view of the axis-symmetric property of the problem (with respect to the rod), we adopt 2-D configuration, as shown in Fig. (1). The formation of lightning channel is divided into two parts: downward leader and upward leader. So our simulation should consider the inception and development of multi upward leaders and the implementation of the final-jump phase of leader process on the basis of fractal simulation of general gas discharge. We randomly choose a point from the upper boundary of the 2-D configuration and generate a section of downward leaders to initiate our simulation. Since the approach of a lightning leader from the thundercloud does not depend on the existence of ground objects until it enters a "striking distance" of structures [4], we only need to make a cutoff at certain height $H$ (in this paper we set $H=500 \mathrm{~m}$ ). The horizontal scale $W=2 \mathrm{~km}$ is so chosen that the downward leaders initiating from places beyond the side boundary will not strike the grounded structures and therefore not contribute to the results in concern. The lower boundary in Fig. (1) represents the ground, in the central area of which we set the model of a lightning rod (and a slender protected structure if necessary). As the downward leader approaches the ground, upward leaders may be initiated from the grounded structures and develop to meet the downward leader.

In the simulation (the mesh is 1000 (vertical) $\times 4000$ (horizontal) and the length unit is $0.5 \mathrm{~m}$ ), the ground and structures on it (like the rod and protected structure) are set to be zero potential while the initiated downward leader tip is assigned potential $-U_{0}$. Then implement the DBM process stated in the previous subsection. At each time step, check the mean field between the head of downward leader and the ground, the lightning will strike to the ground if the field is greater than critical value $E_{g}$ (this is most possible when the initial point of downward leader is close enough to the side boundary). We check the field at points on the grounded objects from where an upward leader may incept. If the mean field between such a point and its neighboring point is greater than a critical value $E_{u}$, breakdown along this direction will happen and upward leader process will begin. Every upward leader that has incepted will develop in the manner similar to downward leaders except that the velocity ratio of upward and downward leaders is about 1:3 according to Ref. [2]. Then we will calculate the mean field between the head of downward leader and the head of every upward leader and suppose the maximum value $E^{(k)}$ corresponds to the $k$ th upward leader. If final-jump does not happen, i. e. $E^{(k)}$ is no more than $E_{f}$ (the critical field for final jump), the simulation will enter the next step; otherwise the developing process of leader channel will end and we can conduct postprocessings, including calculation of fractal dimension and record of lightning strikes. The result of a single simulation obviously has randomness of a high degree. To make the simulation results of practical use, we must conduct simulations iterately and obtain statistical results.

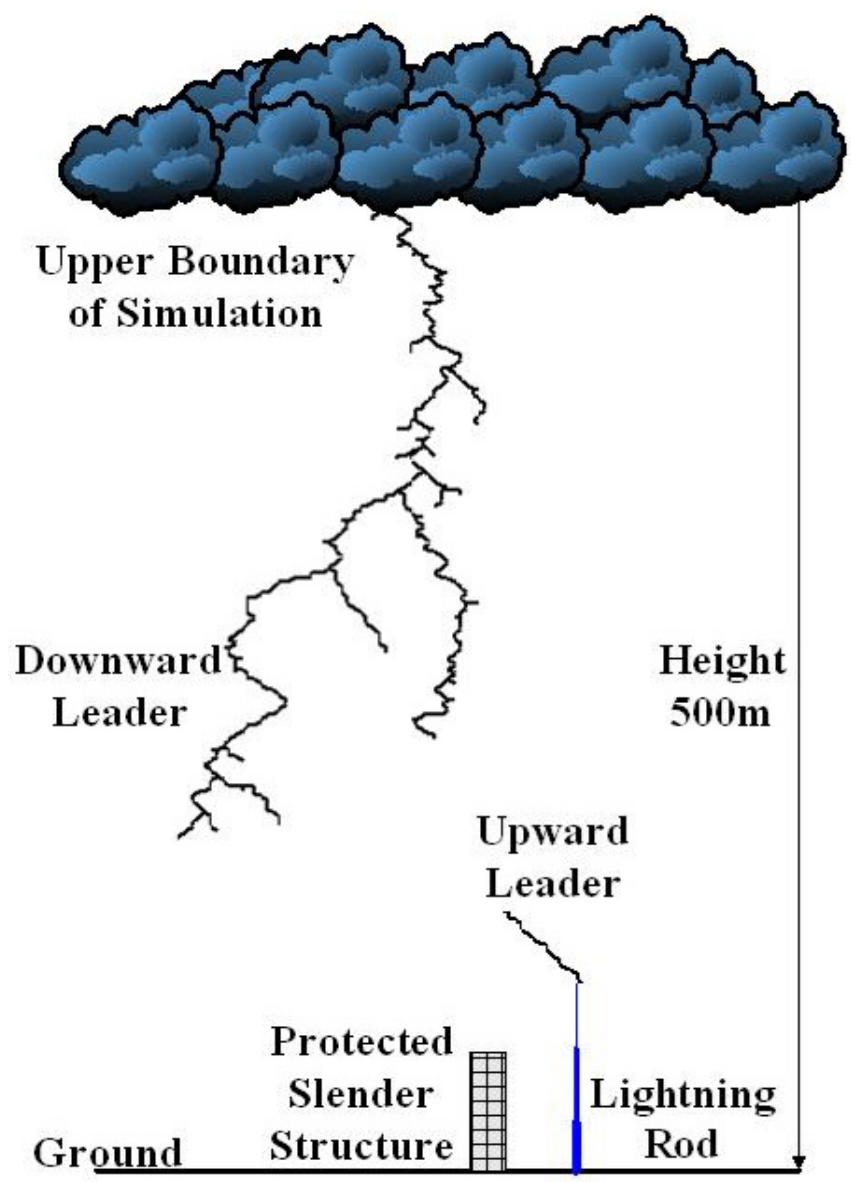

Fig. (1). Configuration for our fractal simulation of lightning strikes to lightning rod. 


\section{Parameter Settings}

The values for $E_{c}$, the critical field for leader propagation and $E_{c h}$, the field along leader channel can be found in some previous works. In our simulation, $E_{c}$ for positive and negative leaders are 5 and $10 \mathrm{kV} / \mathrm{cm}$ respectively $[7,8] . E_{c h}$ is chosen to be $17 \mathrm{kV} / \mathrm{m}$ based on the data provided in Tables 2 and 3 of Ref. [10]. The criterion for final-jump, $E_{f}$, and the critical field of direct strike to the ground, $E_{g}$, is temporarily from estimation, that is, set as part of the breakdown field of a gas gap, typically $15 \mathrm{kV} / \mathrm{cm}$. The criterion for upward leader propagation $E \geq E_{c}$ is not the same as the criterion for upward leader inception. A generally-accepted criterion for positive upward leader inception is that the local field intensity should satisfy $E \geq E_{u} \approx 500 \mathrm{kV} / \mathrm{m}[11]$.

The protective performance of lightning rod is closely related to the lightning current magnitude, which has not yet been included in the simulation. In China, the recommended probability distribution of lightning current magnitude is shown in Eq. (5) [12].

$\log P=-\frac{I}{88}$

where $I_{m}(\mathrm{kA})$ is the lightning current magnitude, and $P$ is the probability of current exceeding $I_{m}$. Assume the initial leader tip potential $\left(U_{0}\right)$ is in proportion with the magnitude of lightning current, i.e., $U_{0}(\mathrm{MV})=Z I_{m}(\mathrm{kA})$, where $Z$ is the coefficient. Obviously, this rough relation is not a universal one, since the values of $U_{0}$ are positively related to the height of the simulation area, $H$. However, in this work we set this coefficient $Z=0.5$ based on the following facts: (a) according to Eq. (5), the probability of current exceeding $200 \mathrm{kA}$ is negligible and thus lightning with $I_{m}$ larger than 200kA is nearly impossible; and (b) to ensure that the upward leaders and the final-jump gaps are only several ten meters long, in our simulation $U_{0}$ should not be greater than $100 \mathrm{MV}$. Hence the probability distribution of the initial leader tip potential comes to be Eq. (6):

$\log P=-\frac{U_{0}}{44}$

where the unit of $U_{0}$ is $\mathrm{MV}$, and $P$ is the probability of the initial leader tip potential exceeding $U_{0}$. By some discretizing procedure, we obtain an approximate probability distribution of $U_{0}$ with discrete values, as shown in Table 1 . And at the beginning of each simulation, a value of $U_{0}$ is randomly chosen subjected to this distribution. By this way, we can evaluate the "overall effect" of lightning with various currents after hundreds of simulations.

Table 1. Probability Distribution of Thundercloud Potential

\begin{tabular}{|c|c|c|c|c|c|}
\hline $\boldsymbol{U}_{\mathbf{0}}(\mathbf{M V})$ & 20 & 30 & 50 & 70 & 100 \\
\hline Probability & 0.45 & 0.31 & 0.14 & 0.08 & 0.02 \\
\hline
\end{tabular}

As a geometrical method, the fractal dimension serves as a criterion to testify the credibility of simulation results. It can be obtained from both photographs of lightning flashes and the channels generated by fractal simulation. Put the lightning channel on a photo into a lattice with boxes of side length $r_{1}$, and count the number of boxes (denoted $N_{1}\left(r_{1}\right)$ ) intersecting with the channel. Lessening the side length to $r_{2}$, the number becomes $N_{2}\left(r_{2}\right)$ and we can keep doing this. The definition of fractal dimension (box dimension) is shown in Eq. (7) [9].

$$
D=\lim _{r \rightarrow 0}\left(\frac{\ln [N(r)]}{\ln (1 / r)}\right)
$$

From Eq. (6) we know $N(r) \sim r^{-D}$. The logarithms $\ln N_{i}$ and $\ln r_{i}$ are fitted into a straight line in double-logarithmic coordinate, the slope of which is the fractal dimension $D$.

The fractal dimension of natural lightning discharge is in an acceptable range from 1.1 to 1.4 [9]. The fractal dimensions calculated from our simulation results vary with the development probability index $(\eta)$, as shown in Table 2 . When $\eta$ is larger, the corresponding fractal dimension is smaller. And we can find $\eta=2 \sim 3$ is the closest to the observational data.

Table 2. Fractal Dimension of Lightning Discharge in Our Simulation (Sample Number: 30)

\begin{tabular}{|c|c|}
\hline$\eta$ & Fractal Dimension $\boldsymbol{D}$ Average (S.D.) \\
\hline \hline 0.5 & $1.89(0.2000)$ \\
\hline 1 & $1.55(0.0636)$ \\
\hline 2 & $1.17(0.0432)$ \\
\hline 3 & $1.10(0.0404)$ \\
\hline 4 & $1.01(0.0320)$ \\
\hline
\end{tabular}

\section{STRIKE DISTRIBUTION AND INTERCEPTION AREA OF A LIGHTNING ROD}

\section{A. Strike Distribution}

Suppose there is only a lightning rod located at the midmost point on the ground. We set hot-spots (the points from where an upward leader can incept, usually located at places with large curvatures such as edges, corners and tips) every several meters along the lightning rod. The strikes to hotspots other than the rod tip are occurrences of lateral strikes on the rod and may contribute to shielding failures if some protected structure exists nearby. We investigate rods with the height of $40 \mathrm{~m}, 60 \mathrm{~m}, 80 \mathrm{~m}$ and $100 \mathrm{~m}$ and conduct 300 iterative simulations for each case to obtain the strike distributions on these rods, as shown in Figs. $(\mathbf{2}, \mathbf{3})$.

In Figs. (2, 3), we use small squares to illustrate the distribution of hot-spots along the rod (in fact, the hot-spots are located along the edge of the 2-D model of the rod). Obviously, the overwhelming majority of lightning will strike the rod tip, which accounts for the constantly wide use of lightning rod. But there is something we do not expect to occur. All the four cases have a common appearance that the upper half section of the rod (except the tip) can be hit at a relatively small probability, which reveals that high lightning rods have inherent vulnerability in protecting structures from lateral strikes. 
․ Hot-spots free from strikes
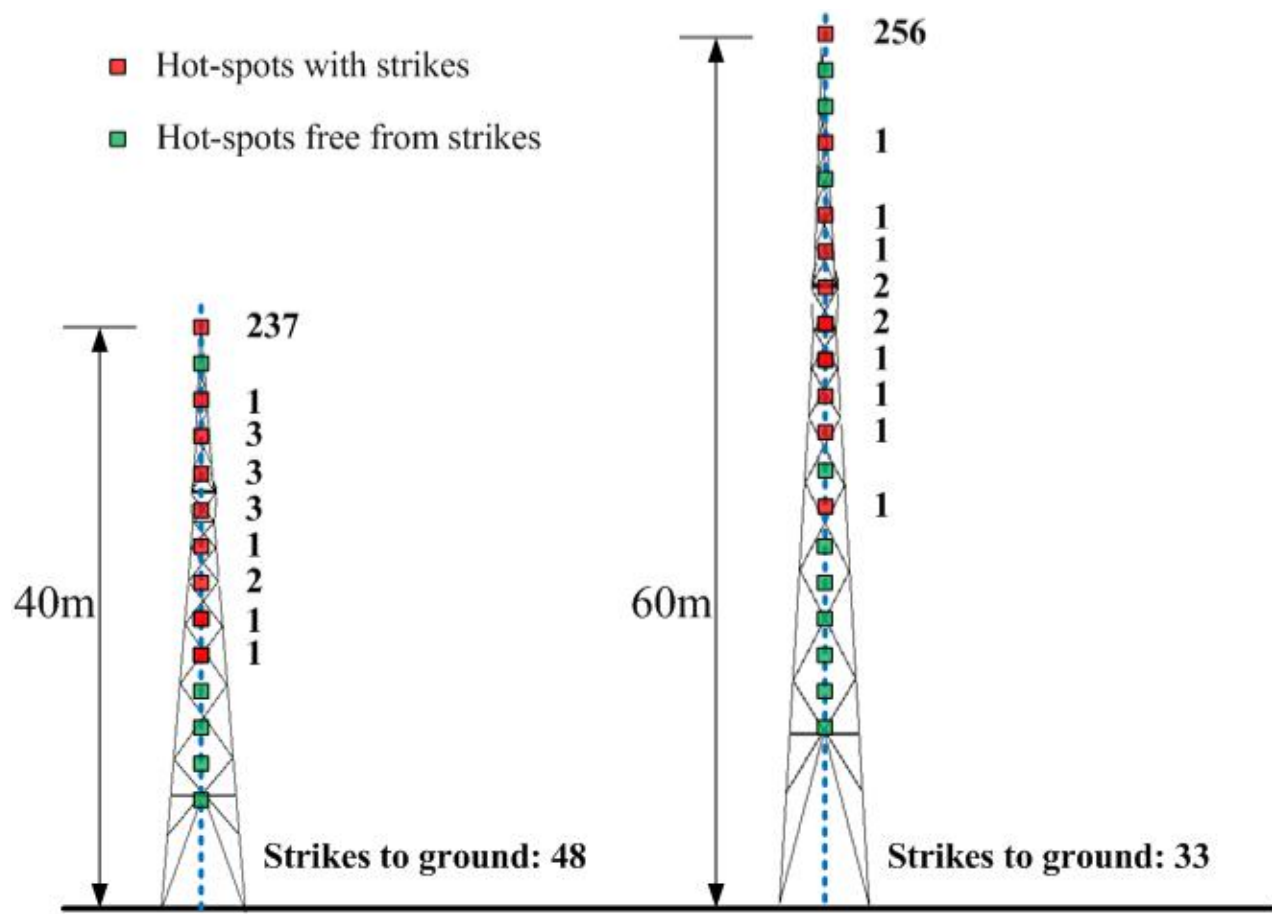

Fig. (2). Distribution of strikes on the rod with height of $40 \mathrm{~m}$ and $60 \mathrm{~m}$.

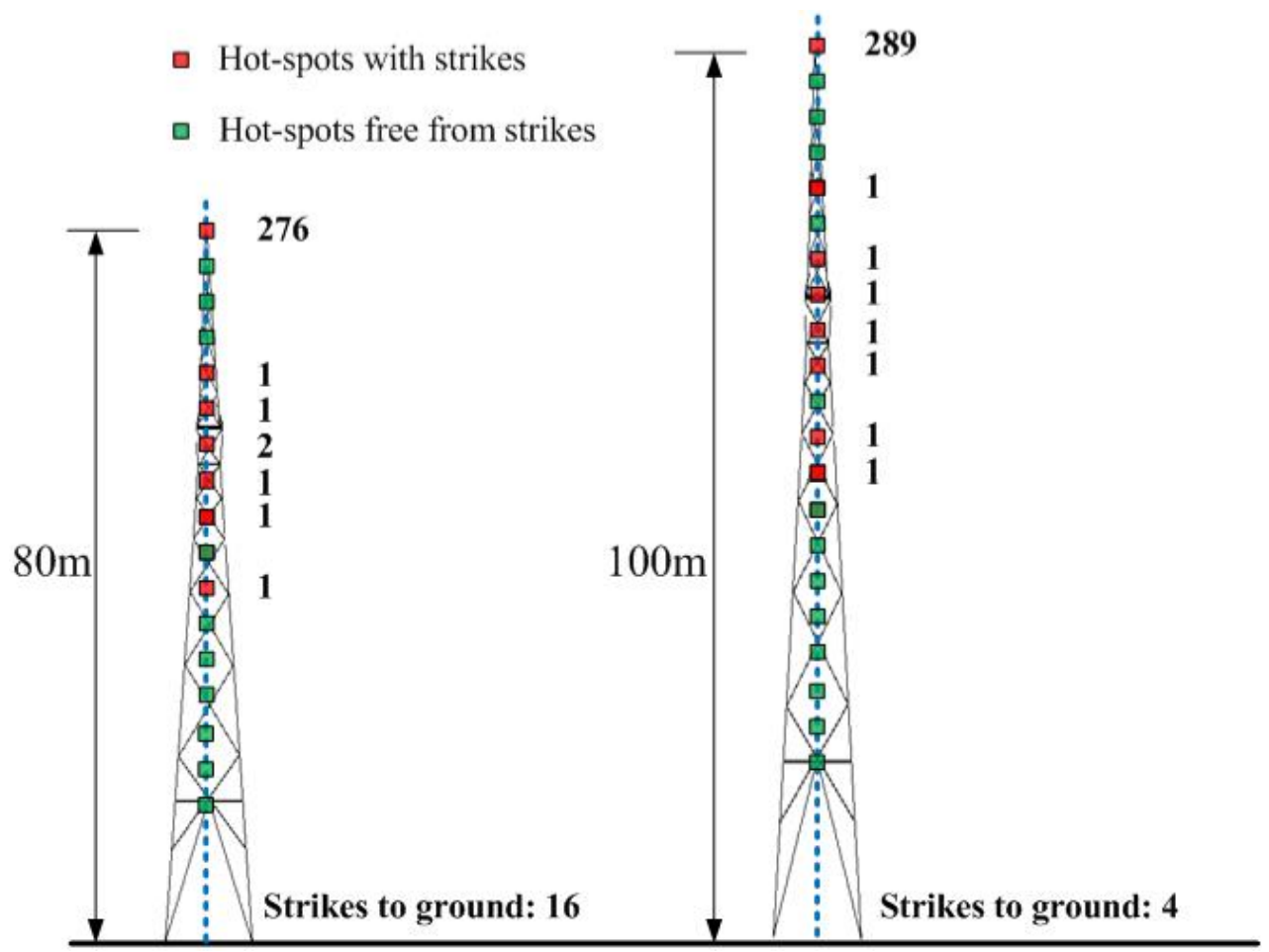

Fig. (3). Distribution of strikes on the rod with height of $80 \mathrm{~m}$ and $100 \mathrm{~m}$.

Several trends can be found in Figs. $(2,3)$ : (1) as the height of the rod increases, strikes to ground would be fewer and fewer due to the growing ability of the rod to intercept the lightning; (2) the lower half of the rod is free from lightning strikes because the height makes upward leaders incepting here not competitive to attach the downward leader; (3) strikes to the place right below the rod tip is rare, since the streamer from the tip has shielding effect like a Faraday's cage, which largely reduces the electric field backward and restricts further development of upward leaders incepting from nearby points; (4) at about the middle part of the rod the strike probability is relatively large, implying that at this height the protection zone of the rod will meet a turning point, as will be shown in Section 4 .

\section{B. Interception Area}

In the above simulations, initial downward leaders approach the rod at different lateral displacements. According 
to Ref. [13], the interception area concept characterizes better the risk or effectiveness of the lightning protection systems than the protected zone concept. And there is an effective interception area $s\left(P_{i}\right)$ on the upper boundary of the configuration as function of the probability of strikes to the tip of the rod, $P_{i}$. Its practical meaning is that if and only if the simulated lightning initiates from the area, it would be caught by the rod tip with the possibility of $P_{i}$. We introduce the scaled effective interception area $A\left(P_{i}\right)$ defined by Eq. (8):

$$
A\left(P_{i}\right)=\frac{s\left(P_{i}\right)^{2}}{W^{2}}
$$

where $W$ is the width of the simulation configuration. The square means the problem is actually 3-dimensional and the horizontal cross section of the interception zone ought to be a circle.

Fig. (4) shows the scaled effective interception area of a single rod with height of $40 \mathrm{~m}, 60 \mathrm{~m}, 80 \mathrm{~m}$ and $100 \mathrm{~m}$. As $P_{i}$, the probability of strikes to the tip of the rod, increases, the scaled effective interception area $A\left(P_{i}\right)$ declines sharply. The higher the rod is, the larger $A\left(P_{i}\right)$ would be. These features are obvious. However, what we concern more are the limit cases.

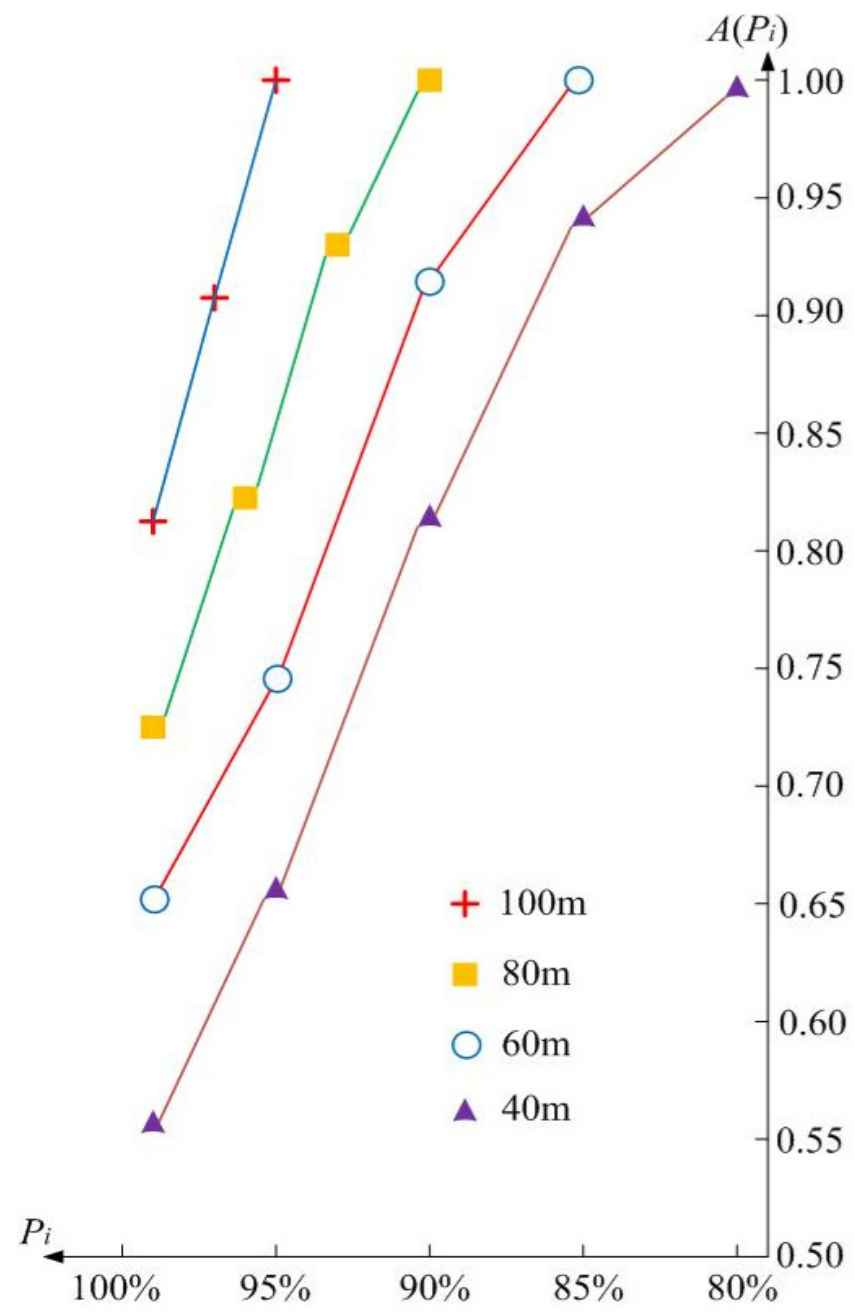

Fig. (4). Scaled effective interception area $A\left(P_{i}\right)$ of single rods with height of $40,60,80$ and 100 meters.
When $A\left(P_{i}\right)$ approaches 1.00 , the corresponding $P_{i}$ approximately represents the probability of lightning caught by the rod tip. In Fig. (4), this probability is about $80 \%, 85 \%$, $90 \%$, and $95 \%$ for lightning rod with the height of $40 \mathrm{~m}, 60$ $\mathrm{m}, 80 \mathrm{~m}$ and $100 \mathrm{~m}$, which displays a linear relation between rod height and the probability of lightning caught by the rod tip.

When $P_{i}$ approaches $100 \%$, the corresponding $A\left(P_{i}\right)$ is just the area initiating lightning leaders that will surely be intercepted by the rod tip. From Fig. (4) we know that this scaled area is $0.555,0.652,0.725$, and 0.810 for lightning rod with the height of $40 \mathrm{~m}, 60 \mathrm{~m}, 80 \mathrm{~m}$ and $100 \mathrm{~m}$, which, roughly speaking, also displays a linear relation between rod height and the area initiating lightning leaders that will surely be intercepted by the rod tip.

In the sense of increasing the effective interception area, the suggestion from our simulation is to use high rods. By using higher rods, the rod tip will catch lightning at higher probabilities. And larger effective interception areas can be acquired. In rolling-sphere method, the upper section of a high rod that exceeds the radius of the rolling-sphere is totally useless, which may require modification according to the above results.

\section{PROTECTION ZONE OF SINGLE LIGHTNING RODS}

\section{A. Algorithm}

To determine the protection zone of a single lightning rod which is isotropic along all horizontal directions, another slender protected structure is included in the configuration. The top of the structure is also set as a hot-spot. The breakthrough probability indicating the degree of shielding failure is evaluated by Eq. (9):

$$
P_{b}=\frac{N_{S}}{N_{S}+N_{R}}
$$

where $N_{S}$ and $N_{R}$ denote the number of strikes to the protected structure and the lightning rod, respectively. Iterative calculations will be conducted as illustrated in Fig. (5).

In Fig. (5), $\Delta L$ charaterizes the resolution of the calculated protection zone, $R$ and $h$ are the height of rod and protected slender structure respectively, $S$ is the horizontal distance between them, $P_{0}$ is the allowable breakthrough probability in the protection zone. We start the calculation with $h=R$ and $S=0$. Then increase $S$ by $\Delta L$ (the structure with the same height as the rod stands just beside the rod) and compute the breakthrough probability $P_{b}$. If $P_{b}$ exceeds the allowable probability $P_{0}$, we decrease the height of the structure and compute again until the breakthrough probability is smaller than $P_{0}$. Then we will further increase the separation $S$ between the rod and the structure by a length unit and begin another round of calculation. We set the terminating condition of the calculation as $S$ over a maximum value $S_{\max }$ or the height of the protected structure being smaller than $h_{\text {min }}=5 \mathrm{~m}$.

\section{B. Results for the 40-m Rod}

The $90 \%$ shielding zone (the allowable breakthrough probability $P_{0}=10 \%$ ) of a lightning rod with height of $40 \mathrm{~m}$ is shown in Fig. (6). When $\Delta L=5 \mathrm{~m}$, due to the discretization, 


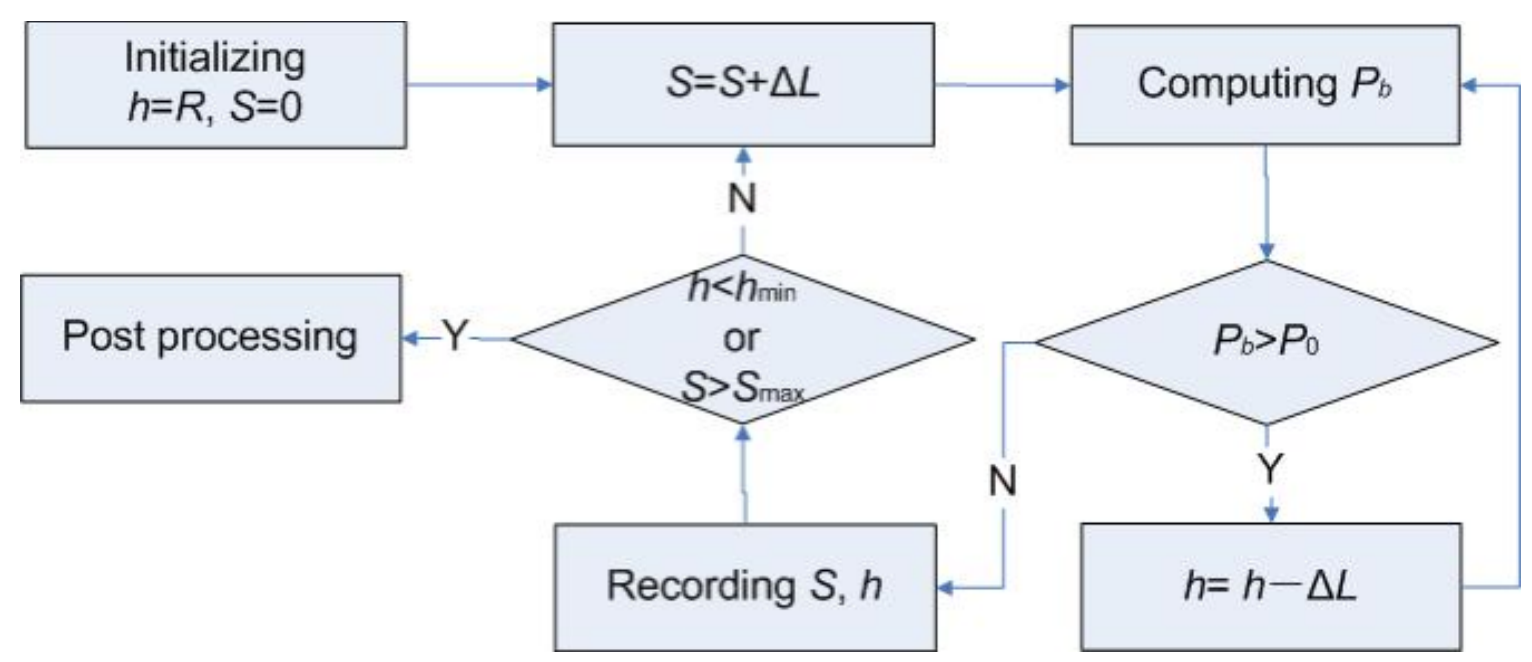

Fig. (5). Flow chart for calculation of protection zone.

the protection zone varies stepwisely instead of displaying a continuous curve. We use the solid line in Fig. (6) to depict the profile. And we only draw the profiles in the following discussion for the sake of simplicity.

The whole protection zone can be divided into three parts: shielding region $\mathrm{A}$, competing region $\mathrm{B}$, and "predominance-of-height" region $\mathrm{C}$. This division is not only based on the slope change of the protection zone profile shown in Fig. (6), but also according to their difference in leader development during our simulations.

In region $A$, streamers may have incepted at the top of the protected structure very near to the rod. But screening effect of the upward leader from the rod disables its further development, so we call this region the shielding area. In region $\mathrm{C}$, though the rod has little interference with protected structures, its predominance of height makes it easy to capture downward leaders. However, in practice, region $\mathrm{C}$ will not extend too much because many other factors will intervene as the distance from the rod becomes large. In region $\mathrm{B}$, upward leaders from both rod and protected structure can propagate and they compete with each other to decide which one satisfies final-jump condition first. Region B is essential in the design of lightning protection. Note that its range of height is just approximate to that of the middle part of the rod. If region B is not well estimated, or the protected structure overtops this region, lateral strikes are more likely to occur.

The $95 \%$ and $99 \%$ protection zones calculated from our fractal simulation are shown in Fig. (7), both of which can also be viewed consisting of three regions. As the allowable breakthrough probability increases (the effectiveness degrades), the region $\mathrm{C}$ tends to expand farther away. We can also note from the $95 \%$ and $99 \%$ protection zones that the improvement of the effectiveness leads to a sharper profile of region A.

We also show in Fig. (7) comparisons between our approach and the rolling sphere method. When the class of lightning protection system (LPS) is I, II and III, the corresponding radius of the rolling sphere is respectively $20 \mathrm{~m}, 30$ $\mathrm{m}$ and $45 \mathrm{~m}$ [1]. The rolling-sphere method is based on two assumptions: (a) the strike point is determined when the downward leader approaches the earth or a structure with a striking distance, and (b) lightning strikes the nearest earthed object from the orientation point and so its worst position is the center of a sphere which attaches several earth objects. As concluded in Ref. [14], no lightning will strike the protected structure if its striking distance is greater than the radius of the sphere. However, in our simulation, it is hard to

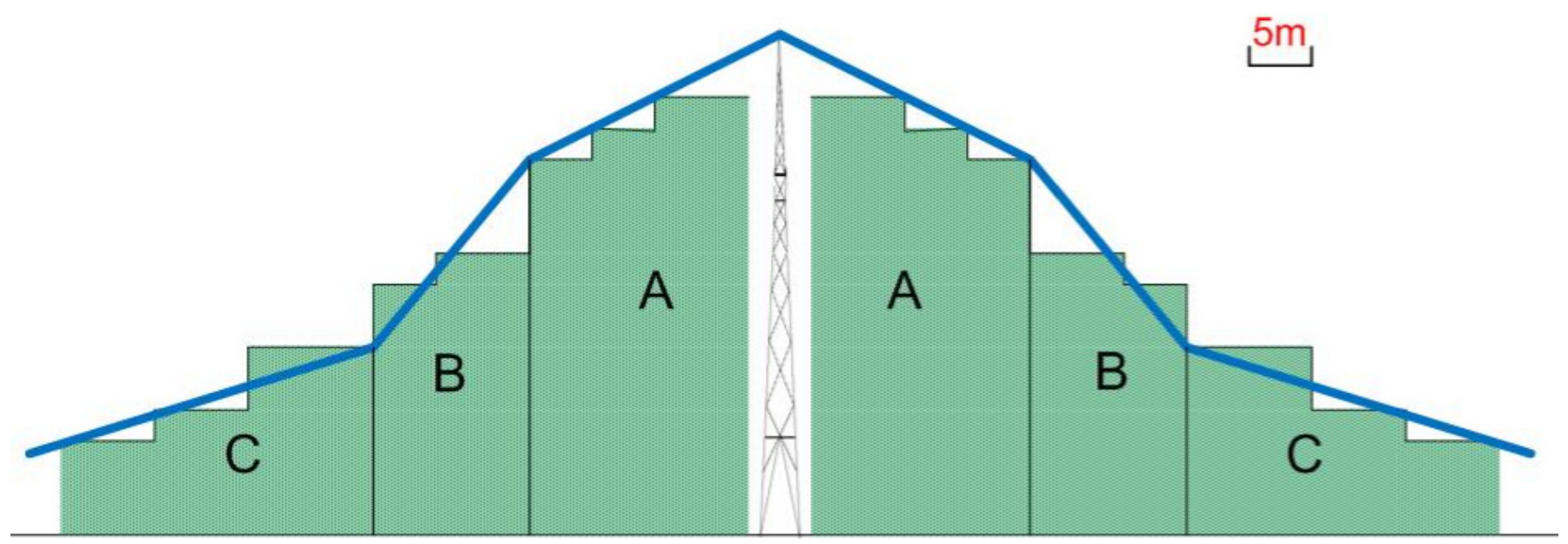

Fig. (6). $90 \%$ protection zone of a 40 -m lightning rod. 


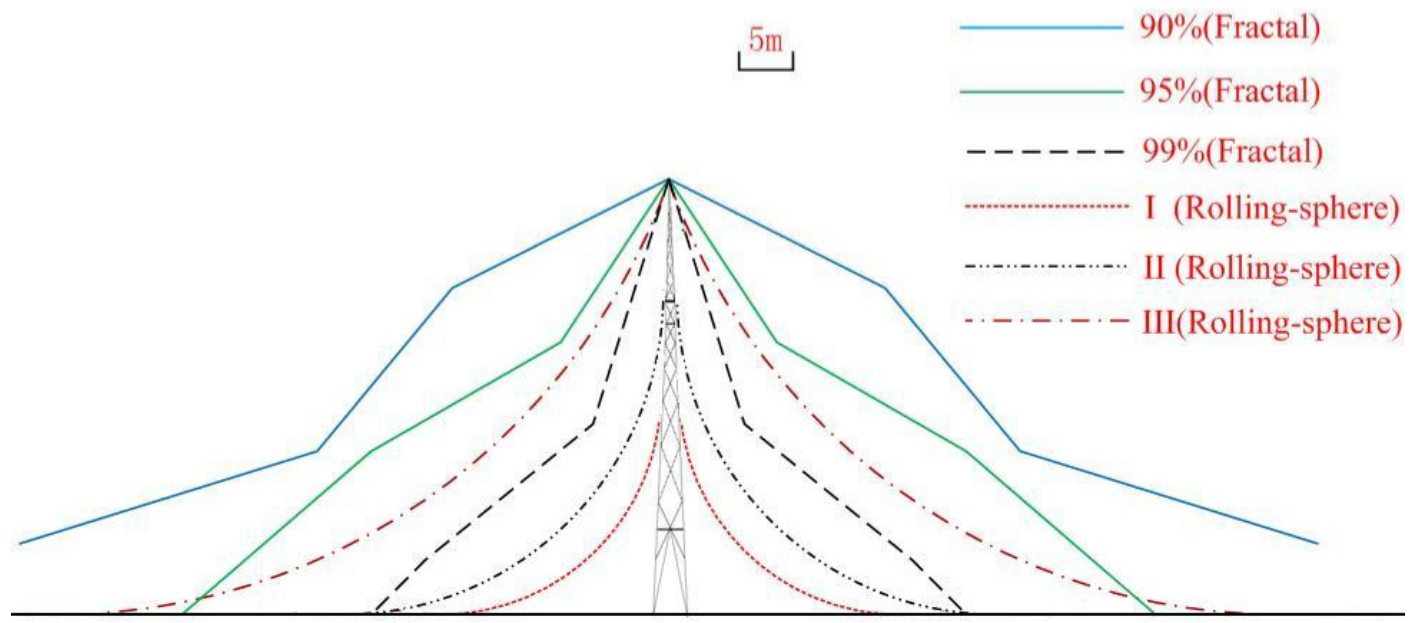

Fig. (7). Protection zones of a 40m lightning rod of different effectiveness and comparisons with rolling-sphere method.

obtain a $100 \%$ protection zone due to the stochastic nature of the model.

As shown in Fig. (7), the 99\% protection zone from fractal simulation is between the results of the rolling sphere method for the Class II and III of LPS. This implies that our fractal approach provide a promising tool for the evaluation of LPS effectiveness (at least for the Class III). Moreover, the fractal method evaluates the performance of lightning protection by means of dynamic simulation, in which the interactions between protected structure and the lightning rod are taken into consideration. It may be complementary to traditional methods in complex configurations. It also adds to our options when designing or evaluating LPS.

\section{More Results}

More cases with different rod heights are simulated, as shown in Fig. (8). No obvious regulations can be found to generate the protection zones of rod with different height in a universal way. Nevertheless, the three regions still exist (due to the interpolation error, the curves corresponding to $100 \mathrm{~m}$ and $80 \mathrm{~m}$ rods look like two sections). It appears that the higher the rod is, the sharper the profile of protection zone turns to be.

The results in Fig. (8) are not the same as that of rollingsphere method. In this method, the protection zone is mainly determined by the radius of the rolling-sphere (which is re-

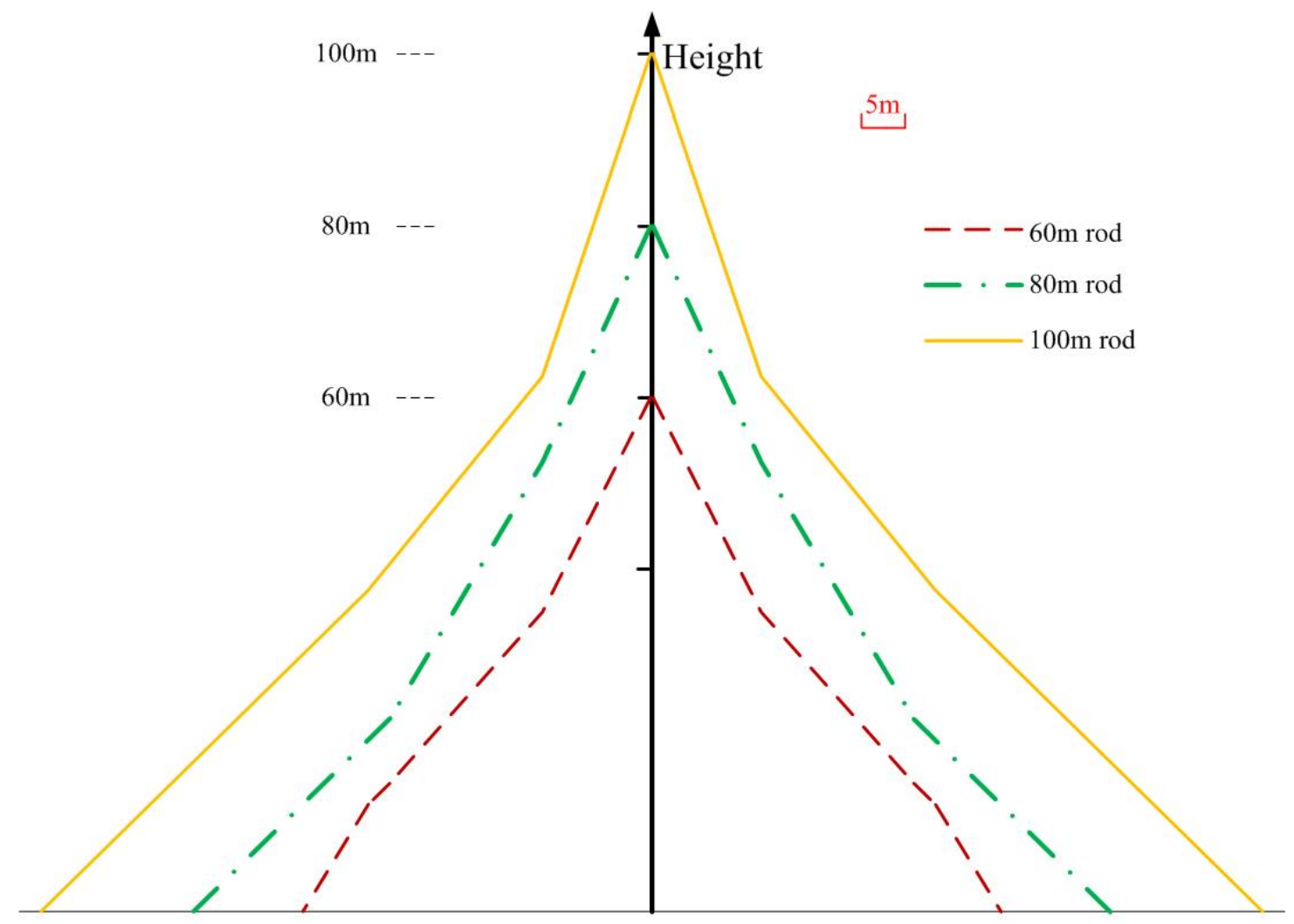

Fig. (8). $95 \%$ protection zones of rod with height of 60,80 and 100 meters. 
lated to the lightning current magnitude) and the increase of the height of rod brings little improvement to the effectiveness. According to Ref. [1], maximum radius of the rollingsphere is $60 \mathrm{~m}$ (Class IV), and the protection zone of a high rod can never be higher than $60 \mathrm{~m}$. However, this is not the case in our simulation. As shown in Fig. (8), the protection zone higher than $60 \mathrm{~m}$ (Region A) is mainly contributed by the Faraday screening effect of the upward leaders incepting from the rod tip.

\section{CONCLUSIONS}

In this paper, we make a series of simulations on every aspect of the effectiveness of single lightning rod based on a 2-dimensional fractal model. Though our simulation results in this paper require lightning observations for further verification, we do provide a powerful method in the lightning protection engineering and explore its potential in practical applications. Our main conclusions are as follows:

(1) The effective interception area increases as the height of the rod augments, analysis results indicate that higher rod tends to catch the lightning more probably;

(2) The strike distribution on the rod (except the tip) is relatively denser on the upper section and thus accounts for the vulnerability of the rod to lateral strikes;

(3) A feature of protection zone of lightning rod named "three regions" are concluded from analysis in cases with different rod height, which shows a different profile compared with traditional methods;

(4) As the height of the rod increases, the profile of the protection zone tends to be sharper and the range of protection zone is not expanding in proportion to the height.

Some improvements will be included in our subsequent works. We find that the simulation results are sensitive to the criterion for the inception of upward leaders and it is necessary to combine the latest upward leader inception model in Ref. $[15,16]$ into our simulation. Also the 3-D fractal simulation should be developed to extend the application of this method to more practical cases.

\section{ACKNOWLEDGEMENT}

This paper is supported by National Nature Science Foundation of China under Grant No. 50737001 and National Basic Research Program of China (973 Program) under Grant No. 2009CB724504.

\section{REFERENCES}

[1] IEC 62305-3 Ed. 2.0, Protection against lightning - Part 3: Physical damage to structures and life hazard, 2006.

[2] Rakov VA, Uman MA. Lightning, physics and effects. Cambridge University Press 2003; pp. 108-42.

[3] Nguyen DT, Deegan G, Alessandro FD. Fractal nature of probabilistic model of lightning discharge. Proc IEEE Reg 10 Int Conf Electr Electron Technol 2001; 2: 814-8.

[4] Petrov NI, Petrova GN, Alessandro FD. Quantification of the probability of lightning strikes to structures using a fractal approach. IEEE Trans Dielectrics Electr Insulation 2003; 10(4): 641-54.

[5] Theodore K, Lin MC. Physically based animation and rendering of lightning. Proc $12^{\text {th }}$ Pac Conf Comput Graph Appl 2004; pp. $267-$ 75.

[6] Takeuti T, Hashimoto T, Takagi N. Two-dimensional computer simulation on the natural stepped leader in summer. J Atmos Electr 1993; 13: 9-14

[7] Dul'zon AA, Lopatin VV, Noskov MD, Pleshkov OI. Modeling the development of the stepped leader of a lightning discharge. Tech Phys 1999; 44: 394-8.

[8] Tsonis AA, Elsner JB. Fractal characterization and simulation of lightning. Atmos Phys 1987; 60: 187-92.

[9] Kawasaki Z, Matsuura K. Does a lightning channel show a fractal?. Appl Energy 2000; 67: 147-58.

[10] Bazelyan EM, Raizer YP. Lightning physics and lightning protection. Institute of Physics Publishing Bristol, England 2000; p. 235.

[11] Dellera L, Garbagnati E. Lightning stroke simulation by means of the leader progression model. IEEE Trans Pow Del 1990; 5 (4): 2023-9.

[12] Chinese Electric Industrial Standard DL/T620-1997, Overvoltage protection and insulation coordination for AC electrical installations, 1997.

[13] Horvath T. Computation of the lightning stroke probability and the effectiveness of the air termination. J Electrostat 1989; 23: 305-22.

[14] Szedenik N. Rolling sphere: method or theory. J Electrostat 2001; 51-52: 345-50.

[15] Becerra M, Cooray V. Time dependent evaluation of the lightning upward connecting leader inception. J Phys D: Appl Phys 2006; 39: 4695-702.

[16] Becerra M, Cooray V. A self-consistent upward leader propagation model. J Phys D: Appl Phys 2006; 39: 3708-15. 